\title{
Pseudo 2-Dimensional Trajectory of a Particle in Vibrating Granular Bed
}

\author{
Panupat Chaiworn, Fei Fang Chung, and Sy-Sang Liaw
}

\begin{abstract}
We experimentally study how the convection patterns of the pseudo two-dimensional granular systems under vibration are affected by the friction between the granules and the side walls of the container. We show that the number of convection cells and the direction of granule velocity are strongly dependent on the magnitudes of the friction. These are adjusted in our experiments by changing the surface property of the container walls, wall motion, and granular height. The larger the friction between the granules and the side wall, the larger the convection cell it generates, and consequently the fewer number of cells the system has. The friction-dependent property of the convection cells is unique to granules not shared by fluid.
\end{abstract}

PACS numbers: 45.70-n, 05.65.+b, 47.20.Bp

Index Terms-Granular system, granular convection, vertical vibration and rectangular container.

\section{INTRODUCTION}

Granular material compacted in a container forms a strong structure like a bulk solid that can afford high pressure at its surface [1]. However, if the container is subjected to a vertical vibration, solid-fluid transition normally occurs [2]. The material becomes easily deformed by shear force and convective flow is started [3]. Particles near the side walls move downward and then rise in the middle of the container (Fig. 1). This convective flow has been studied extensively in literature [4]-[9]. It plays crucial role in many interesting granular topics such as heaping and segregation. Particles carried by the upward flow can stack up to form a heap until a critical slope is reached [10]-[12]. Also, the upward flow is capable to bring large and heavy particle from bottom to the top. Due to the small thickness of the downward flow near the side wall, large particle could not re-enter to the bed. Thus, particles of different sizes in a binary mixture are eventually segregated under vibration [13]-[15]. Apparently, the convection pattern affects strongly the formation of heap [16] and the rise time of large particle [17].

Convective motion occurs in fluid because the instability due to gravity is triggered when the Rayleigh number (or temperature) is larger than a certain value. Granular convection, on the other hand, is induced by a different

Manuscript received October 24, 2013; revised January 13, 2014.

Panupat Chaiworn and Fei Fang Chung are with the Laboratory of Nonlinear Physics, Department of Physics, Chiang Mai Rajabhat University, 50300, Thailand (e-mail: P.Chaiworn@gmail.com, cikgufly001@livemail.tw).

Sy-Sang Liaw is with Center of Nonlinear Physics, Department of Physics, National Chung Hsing University, 402, Taiwan (e-mail: liawsysang@gmail.com). mechanism. It is generally surmised that it is the friction between the granules and the container walls that drags down the particles near side walls to initiate the convection motion. In this report we study explicitly how granular convection patterns depend on the friction by varying friction force in a few different ways. We will see that the number of convection cells is also determined by the friction force.

\section{EXPERIMENTAL SETUP}

The experimental system for this study consists of pseudo-2D rectangular container with thickness fixed at 0.3 $\mathrm{cm}$. The container is adjustable in width $L$, ranging from 3.0 to $12.0 \mathrm{~cm}$. The container is filled with glass beads of diameter $d=0.2 \mathrm{~cm}$ and density $\rho=2.77 \mathrm{~g} / \mathrm{cm}^{3}$ to a height $H=$ $1-5 \mathrm{~cm}$. A sinusoidal signal was used to drive the system to vibrate vertically at frequency $10 \leq f \leq 40 \mathrm{~Hz}$ and amplitude $0.13 \leq A \leq 0.38 \mathrm{~cm}$. For the acceleration we used $(1.56 \leq \Gamma / g \leq 8.85$, where $g$ is the acceleration of gravity), granular convections were clearly observed. We changed the friction force between the particles and the walls by covering the walls with sandpapers of different roughness. Three sandpapers with 32, 40 and 220 particles/inch were used. We have also let one wall move with the vibration and the other remains fixed to see how the convection patterns were affected.

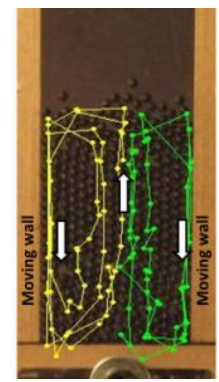

Fig. 1. Granular convection in a pseudo-2D rectangular container normally has two symmetric rolls. Particles go down along the walls and up in the middle (Container width $L=3 \mathrm{~cm}, f=20 \mathrm{~Hz}, A=0.34 \mathrm{~cm}, \Gamma=5.47 \mathrm{~g}$ ).

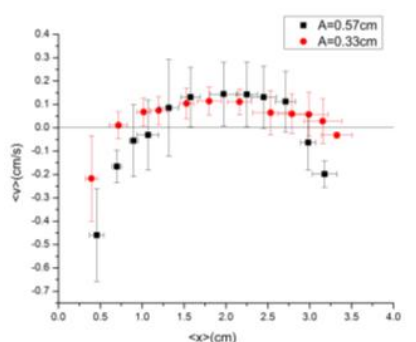

Fig. 2. Average upward speed of the particles as a function of horizontal position in the pseudo-2D rectangular container with both walls moving the external vibration $(f=20 \mathrm{~Hz}$, and $L=3 \mathrm{~cm})$. 


\section{A. Symmetric Walls}

We first measured the average velocities of the particles at half surface height [18] and plotted it as a function of horizontal position across the container (Fig. 2). For a symmetric rectangular container, we see that the particles near side walls move downward and particles in the middle move upward as expected. Apparently, the walls drag beads in contact with them by friction mostly at the stage when the bottom plate is accelerated downward so that the beads near side walls move downward relative to the beads in the middle. If we compare the average upward velocities of particles in the middle under different vibration frequencies and accelerations (Fig. 3), we see that the convective motion is stronger for stronger vibration (which has larger acceleration). Interestingly, the magnitude of the average velocity is in general smaller for larger frequency at any fixed acceleration.

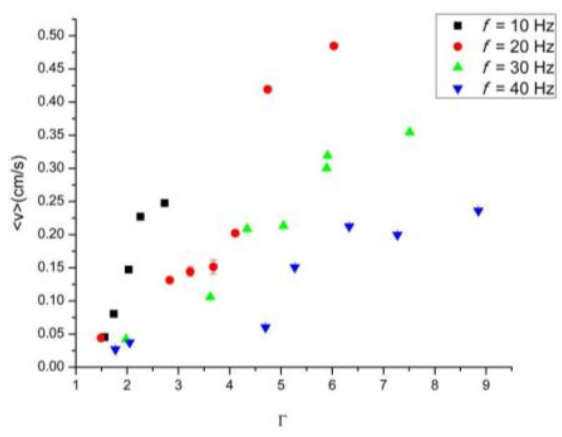

Fig. 3. The average upward speed of particles in the middle of the container increases with acceleration $\Gamma$ at a given frequency $f$. On the other hand, it decreases with $f$ at a fixed $\Gamma(L=3 \mathrm{~cm})$.

\section{B. One Wall Fixed}

We have generated two convection rolls roughly symmetric with respect to the center of the rectangular container whose two side walls are all moving with the external vibration. Next we keep one side wall fixed in the lab coordinates and the other moving with the external vibration. When the bottom plate is accelerated upward, the fixed side wall has larger relative velocity than the movingside wall to its nearby beads so that we expect that the downward friction force on the beads would be larger for those near the fixed wall than those near the other. Consequently, the convection rolls driven by these friction forces would not be symmetric. It turned out in our setup, the difference between friction forces at two side walls is so large that only one convection was observed (Fig. 4 a) ).

\section{Effect of Sandpapers}

On the other hand, one can increase the friction between the beads and the wall simply by pasting sandpaper onto the wall. The static friction coefficient between the bead and the acrylic plate was measured to be 0.24 , while for plate covered with sandpapers of the type with 220, 40 and 32 particles/inch, the friction coefficients were to be $0.30,0.72$, and 0.81 respectively. For example, with one side wall covered with sandpaper of the type with 32 particles/inch, we obtained one convection roll only (Fig. 4 b) ), similar to the case when one side wall is kept fixed (Fig. 4 a) ). Now suppose the right side wall is fixed and the left side wall is moving with the vibration. We measured the average upward velocity of the beads near the left side wall. The speed is positive as we have seen. We then pasted the left side wall with sandpapers, the average upward velocity changes to be negative (Fig. 5) which means the friction effect due to sandpapers is larger than fixed wall. Fig. 5 also shows that the rougher the sandpaper is, the larger the downward speed.
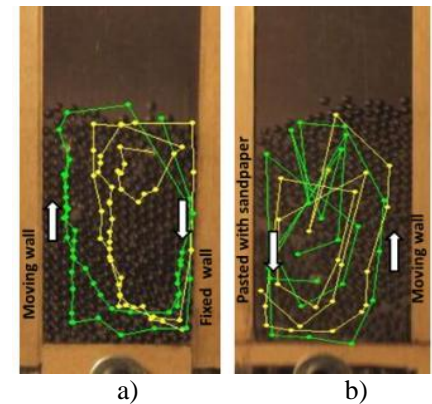

Fig. 4. Asymmetric condition would generate single convection roll: a) When the right wall is fixed while the left wall moves with the external vibration, particles go down along the right wall. b) When the left wall is pasted with sandpaper, particles go down along the left wall. $(L=3 \mathrm{~cm}, f=20 \mathrm{~Hz}, A=$ $0.34 \mathrm{~cm}, \Gamma=5.47 \mathrm{~g}$ ).

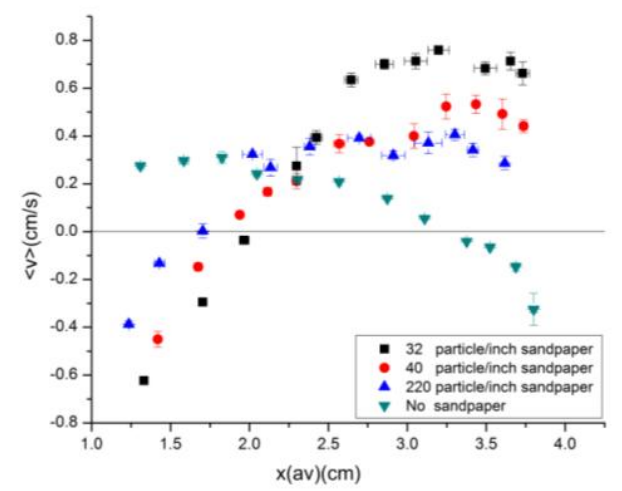

Fig. 5. Average upward speed of particles as a function of horizontal position in the container $(L=3 \mathrm{~cm}, f=20 \mathrm{~Hz}$.)Left wall is fixed while right wall moves with the vibration. Particles go down along the right wall. When sandpapers are pasted on the left wall, particles go down along the left wall.

\section{Effect of Container Length}
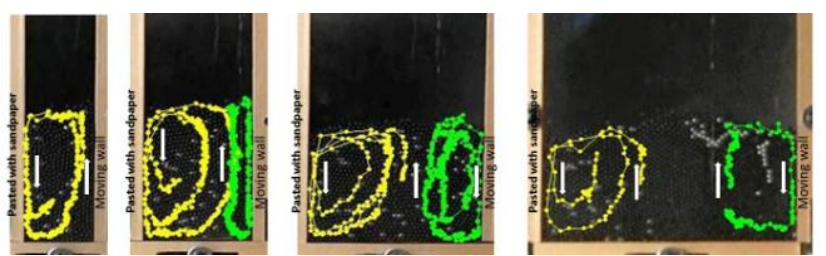

Fig. 6. Effects of the container length. Both walls are moving with vibration and the left wall is pasted with sandpaper. The container width is $L=3 \mathrm{~cm}, 5$ $\mathrm{cm}, 8 \mathrm{~cm}$, and $11 \mathrm{~cm}$ respectively $(f=20 \mathrm{~Hz}, A=0.34 \mathrm{~cm}, \Gamma=5.47 \mathrm{~g})$.

We have seen that there is only one convection roll for the case of two moving side walls with sandpaper on one of them (Fig. 4 b) and Fig. 6 a) ). When we increased the container length (i.e., the distance between the two side walls) from $L=$ $3 \mathrm{~cm}$ to $L=5 \mathrm{~cm}$, we saw a small convection roll developed close to the right side wall (Fig. 6 b) ). In a longer container with length $L=8 \mathrm{~cm}$, both convection rolls grew in size (Fig. $6 \mathrm{c})$ ), with left one larger than the right one. Increasing the length further, we saw that neither rolls grew (Fig. 6 d) ), but instead, two small convection rolls developed between them (not shown in Fig. 6). This showed that the driving force for 
the convection roll was indeed coming from the friction between the beads and the side walls. This friction force gave horizontal momentum for beads at foot of the side walls. These beads moved horizontally toward the other side until their momentum vanished or was balanced by the opposing momentum before turned upward.

\section{E. Effect of Surface Height}

The height of the granular bed affects friction too. The higher the granular surface, the larger the friction is because there are more beads along the wall being dragged downward Consequently, beads gain stronger horizontal momentum so that the convection cells have longer lateral length. In a container of fixed length, this means there is less number of convection cells when system has higher granular height. In Fig. 7 we decreased the granular height in otherwise the same system and indeed observed the number of convection rolls increased.

a)

b)

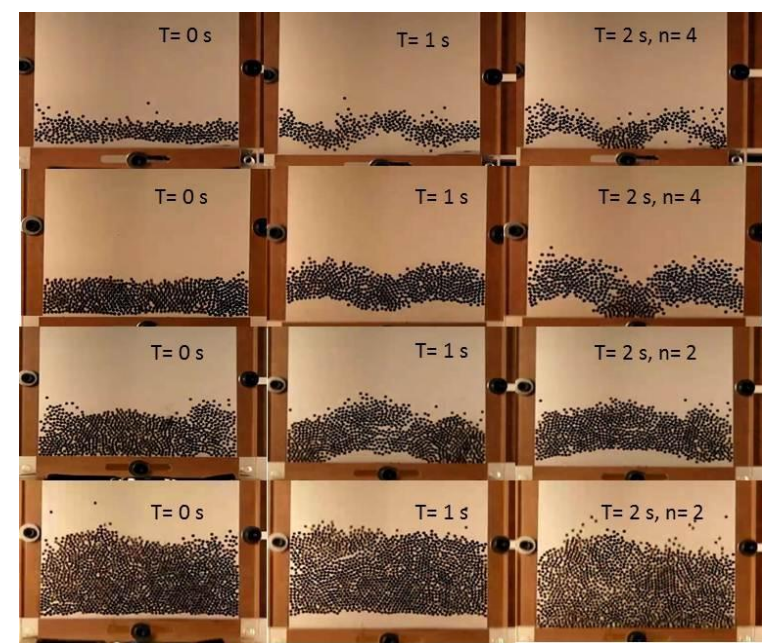

Fig. 7. Snapshots of granular convection patterns. Initial granular height is a) $H=1 \mathrm{~cm}$;b) $H=2 \mathrm{~cm}$; c) $H=2.5 \mathrm{~cm}$; d) $H=5 \mathrm{~cm}$. The value of $n$ indicates the number of convection rolls. $(\varepsilon \approx 0.24, f=20 \mathrm{~Hz}, A=0.62 \mathrm{~cm}, \Gamma=10.06 \mathrm{~g}$, $L=11 \mathrm{~cm}$ ).

\section{F. Friction and the Number of Convection Cells}

a)

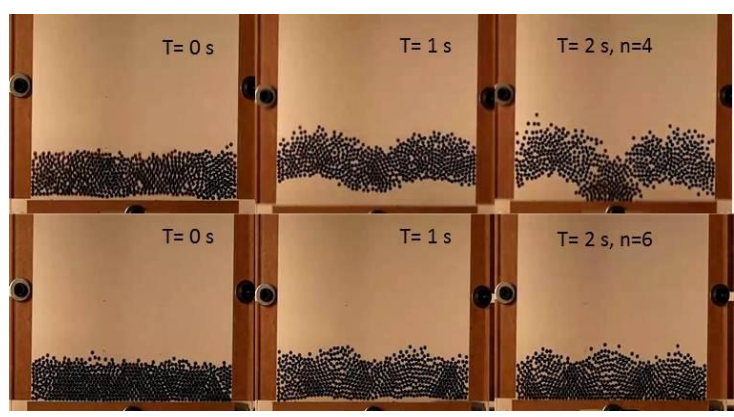

Fig. 8. Effects of vibration acceleration. a) $\Gamma=10.06 \mathrm{~g}$. b) $\Gamma=5.00 \mathrm{~g}$. $(f=20 \mathrm{~Hz}$, $L=11 \mathrm{~cm}$, and $H=2 \mathrm{~cm}$.)

a)

b)

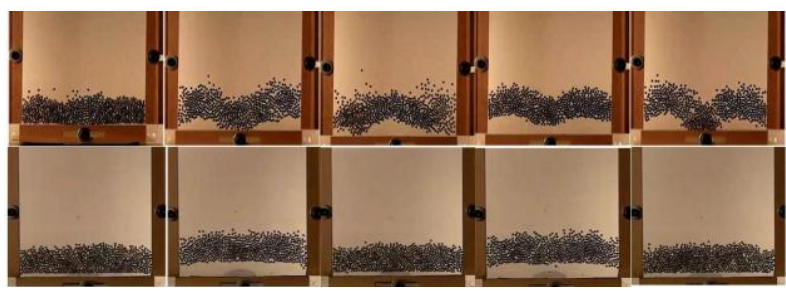

Fig. 9. Effects of friction coefficients. a) $\varepsilon \approx 0.24$ (no sandpaper) b) $\varepsilon \approx 0.81$ (with sandpaper). ( $f=20 \mathrm{~Hz}, A=0.62 \mathrm{~cm}, \Gamma=10.06 \mathrm{~g}, L=11 \mathrm{~cm}$ ).
Above experimental results have indicated that the larger the friction between the particles and the side walls, the less the number of the convection cells. Figs. 8 and 9 are the results of another two experiments in consistent with this indication. In Fig. 8 we compared the results for two systems having different roughness for the side walls. The one pasted with sandpapers has larger friction so that generates less number of convection cells. In Fig. 9 we compared the results of a system under two different accelerations of the vibration. The one with small acceleration has smaller friction so that generates more number of convection cells. Note that Ref. [7] had reported a result showing that the number of convection rolls in the rectangular containers increases with vibration acceleration, apparently opposite to our results. They operated their system at large accelerations $(\Gamma>6 \mathrm{~g})$ and generated the reversed convection rolls where particles went down in the middle and rose up along the side walls. These reversed convections are not driven by the friction between the particles and the walls as we described above so that our argument is not applicable to them. It is interesting to know the mechanism behind the reversed convections in the future.

\section{G. One Shorter Wall}

We have seen that the more number of beads in contact with the side wall the larger the friction is to generate downward momentum, yielding a larger convection cell. We did another experiment to demonstrate this point. In a rectangular container we inserted a bar from top in the middle without touching the bottom (Fig. 10). We expect that the friction produced by this central bar on dragging the beads is smaller than that by the side wall because there is less number of beads in contact with the bar. As one can see from Fig. 10, the convection roll generated by the central bar is indeed smaller than the one by the side wall.
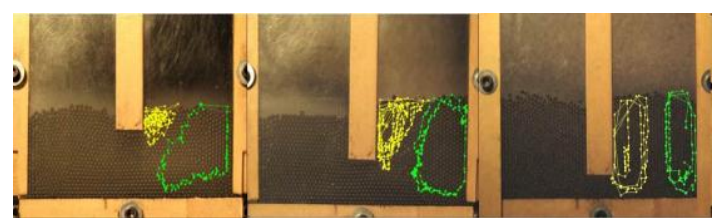

Fig. 10. Trajectories of a bed particle in the pseudo-2D rectangular container. The inner bar is raised above the bottom by a height $3.0,1.5,1.0$, and $0 \mathrm{~cm}$, respectively. $(H=4 \mathrm{~cm}, f=20 \mathrm{~Hz}, A=0.33 \mathrm{~cm}, \Gamma=5.31 \mathrm{~g})$.

\section{CONCLUSION}

In conclusion, we have done a series of experiments to find the relationship between the friction and the convection cells for granular particles contained in a rectangular container under vertical vibration. Under small vibration, the friction between the particles and the walls will drag down the particles along the side walls so that initiate the convection rolls. If the friction is large enough, two symmetric convection rolls appear and particles rise to the surface in the middle of the container. Pasting sandpaper on one side wall will increase the size of the convection roll on the side with sandpaper and force the other smaller. If we then decrease the friction by decreasing the initial granular height or the vibration acceleration, both convection rolls reduce their sizes and between them another two small convection roll occur. From these results and others described in text, we 
conclude that at small vibration, the friction between the particles and the walls is the key factor that determines the sizes and the number of the granular convection rolls in a container, while the friction increases with the roughness of the wall, the granular height, and the vibration acceleration.

\section{ACKNOWLEDGMENT}

This work was supported by grants from the National Science Council under grant number NSC97-2112-M005-001, and the National Center for Theoretical Sciences of Taiwan.

\section{REFERENCES}

[1] H. M. Jaeger, S. R. Nagel, and R. P. Behringer, Rev. Mod. Phys., vol. 68, no. $1259,1996$.

[2] P. Evesque, E. Szmatula, and J.-P. Denis, Eur. Phys. Lett., vol. 12, pp. 623-627, 1990.

[3] Y-H. Taguchi, Phys. Rev. Lett., vol. 69, pp. 1367, 1992.

[4] J. B. Knight, E. E. Ehrichs, V. Y. Kuperman, J. K. Flint, H. M. Jaeger, and S.R. Nagel, Phys. Rev., vol. 54, pp. 5726, 1996

[5] J. B. Knight, Phys. Rev. E, vol. 55, pp. 6016, 1997.

[6] J. A. C. Gallas, H. J. Herrmann, and S. Sokolowski, Phys. Rev. Lett., vol. 69, pp. 1371, 1992.

[7] K. M. Aoki, T. Akiyama, Y. Maki, and T. Watanabe, Phys. Rev. E, vol. 54, pp. 874, 1996.

[8] P. Eshuis, D. van ver Meer, M. Alam, H. J. van Gerner, K. van der Weele, and D. Lohse, Phys. Rev. Lett., vol. 104, pp. 038001, 2010

[9] A. Garcimartin, D. Maza, J. L. Ilquimiche, and I. Zuriguel, Phys. Rev. E, vol. 65, pp. 031303, 2002.

[10] P. Evesque and J. Rajchenbach, Phys. Rev. Lett., vol. 62, pp. 44, 1989.

[11] E. Clement, J. Duran, and J. Rajchenbach, Phys. Rev. Lett., vol. 69, pp $1189,1992$.

[12] H. J. van Gerner, M. A. van der Hoef, D. van der Meer, and K. van der Weele, Phys. Rev. E, vol. 76, pp. 051305, 2007

[13] J. B. Knight, H. M. Jaeger, and S. R. Nagel, Phys. Rev. Lett., vol. 70, $3728,1993$.
[14] J. Duran, T. Mazozi, E. Clement, and J. Rajchenbach, Phys. Rev. E, vol. 50, pp. 5138, 1994.

[15] D. A. Huerta et al., Phys. Rev. Lett., vol. 92, pp. 114301, 2004.

[16] R. J. Milburn, M. A. Naylor, A. J. Smith, M. C. Leaper, K. Good, M. R. Swift, and P. J. King, Phys. Rev. E, vol. 71, pp. 011308, 2005.

[17] M. E. Mobius, X. Cheng, P. Eshuis, G. S. Karczmar, S. R. Nagel, and H. M. Jaeger, Phys. Rev. E, vol. 72, pp. 011304, 2005.

[18] We traced the beads which are initially located at half surface height for one second and calculated the average upward speeds of these beads within these one second. The speed of camera is 600 frames per second.

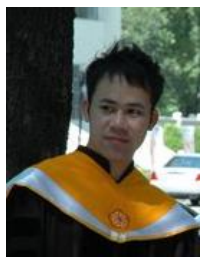

Panupat Chaiworn received his $\mathrm{Ph}$. D. He is in physics from National Chaung- Hsing University, Taichung, Taiwan. $\mathrm{He}$ is currently a lecturer at Chiang Mai Rajabhat University, Chiang Mai, Thailand. His current research interests include granular physics and nonlinear physics.

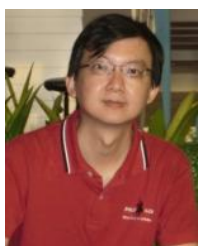

Fei Fang Chung is a lecturer in New Era College Malaysia. He obtained his M. Sc. in physics from National Tsing Hua University and $\mathrm{PhD}$ in Physics from National Chung Hsing University, Taiwan. His interests include Method of computer simulation for granular material and statistics physics.

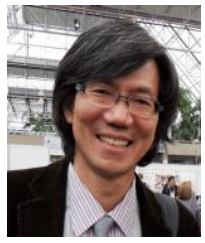

Sy-Sang Liaw is a professor of physics, National Chung-Hsing University, R.O.C. His Ph.D. in Physics, Johns Hopkins University, U.S.A. His current research interests include nonlinear physics, econophysics and biophysics. 